\title{
Influence of integration formulations on the performance of the fast inertial relaxation engine (FIRE) method
}

\author{
Fei Shuang ${ }^{\mathrm{a}, \mathrm{b}}$, Pan Xiao $^{\mathrm{a}, *}$, Ronghao Shi ${ }^{\mathrm{a}, \mathrm{b}}$, Fujiu Ke ${ }^{\mathrm{c}}$, Yilong Bai ${ }^{\mathrm{a}}$ \\ ${ }^{a}$ LNM, Institute of Mechanics, Chinese Academy of Sciences, Beijing 100190, China \\ ${ }^{\mathrm{b}}$ School of Engineering Sciences, University of Chinese Academy of Sciences, Beijing 100049, China \\ ${ }^{c}$ School of Physics and Nuclear Energy Engineering, Beihang University, Beijing 100191, China
}

\section{A R T I C L E I N F O}

\section{Keywords:}

Energy minimization

Fast inertial relaxation engine method

Integration formulations

Molecular simulations

\begin{abstract}
A B S T R A C T
As a simple and robust minimization algorithm with high efficiency, the fast inertial relaxation engine (FIRE) method has been widely applied in various fields. Different from the viewpoint of previous reports, the present study found out that integration formulations in the FIRE method have huge influence on its convergence performance and capability of critical analysis. Specifically, the Forward Euler (FE) integration is found ill-suited for all the trial applications due to the outdated velocity update; the Velocity Verlet (VV) integration shows robust convergence and superior efficiency, but loses the ability of critical analysis; the Semi-implicit Euler (SE) integration endows the FIRE method with the critical analysis ability as well as good efficiency, but its convergence is conditional. It is also found that the FIRE method using a modified energy monitor shows more robust convergence than using the original power monitor. Further investigation indicates that the SE integration combined with the energy monitor should be the first choice for the FIRE method in general molecular statics simulations. These findings extend the capability of FIRE and provide practical suggestions for selecting minimization algorithms in molecular simulations.
\end{abstract}

\section{Introduction}

Energy minimization (EM) of atomistic systems is one of the most common tasks in computational material sciences [1], solid-state physics [2], chemistry and biology [3]. EM is also the core algorithm in many simulation methods, such as molecular statics (MS) [4], coarse-grained methods [5] and recently developed multi-scale methods [6]. Improving the efficiency of EM, therefore, is of great importance to reduce the computational cost in simulations. A variety of well-established mathematical optimization methods, like the steepest decent (SD), conjugate gradient (CG) and quasi-Newton method have been widely used $[7,8]$. When combined with multigrid method, EM shows the superior efficiency in simulations of elastic deformation [9] and dislocation relaxation $[10,11]$. Some other EM methods are variants of molecular dynamics (MD) and have been applied in MS simulations, such as QuickMin (QM) [12] and Fast Inertial Relaxation Engine (FIRE) [13]. Among the MD-like methods, it is reported that FIRE is significantly faster than standard implementations of CG method and often competitive with the more sophisticated quasi-Newton schemes typically in ab initio calculations. The robustness and versatility in critical point analysis also make FIRE more intriguing than other EM methods.
Due to its high efficiency, simplicity and low computational consumption, FIRE has been implemented as an effective minimizer into various simulation packages, such as LAMMPS [14], HOOMD-blue $[15,16]$, BigDFT [17] and EON [18], which play an important role in the frontier research of material sciences. Rogan et al. adopted FIRE as an efficient local minimizer to search for the global and local minimal energy states of freestanding nanoclusters [19]. Fayon et al. utilized FIRE to optimize geometry in HOOD-blue to study the formation mechanism of ultra-porous framework materials [20]. Hwang et al. used FIRE to study bubble super-diffusion and soft glassy materials [21]. As emphasized by Bitzek [13], integration formulation has little influence on the FIRE method. However, our preliminary results indicate that FIRE with the default integration formulation is much less efficient than CG in LAMMPS package, which is inconsistent with the conclusion in aforementioned work. Recently, a new structure relaxation algorithm based on micro-canonical ensemble (NVERE) proposed by Yang et al. is reported faster than FIRE in relaxing soft structures like graphene sheet [22], which implies that FIRE is still worth further exploring in different applications. Here in this work, we are focusing on the influence of integration formulation on the performance of FIRE so as to extend its capability and provide practical suggestions on general applications.

\footnotetext{
* Corresponding author.

E-mail address: xiaopan@lnm.imech.ac.cn (P. Xiao).
} 


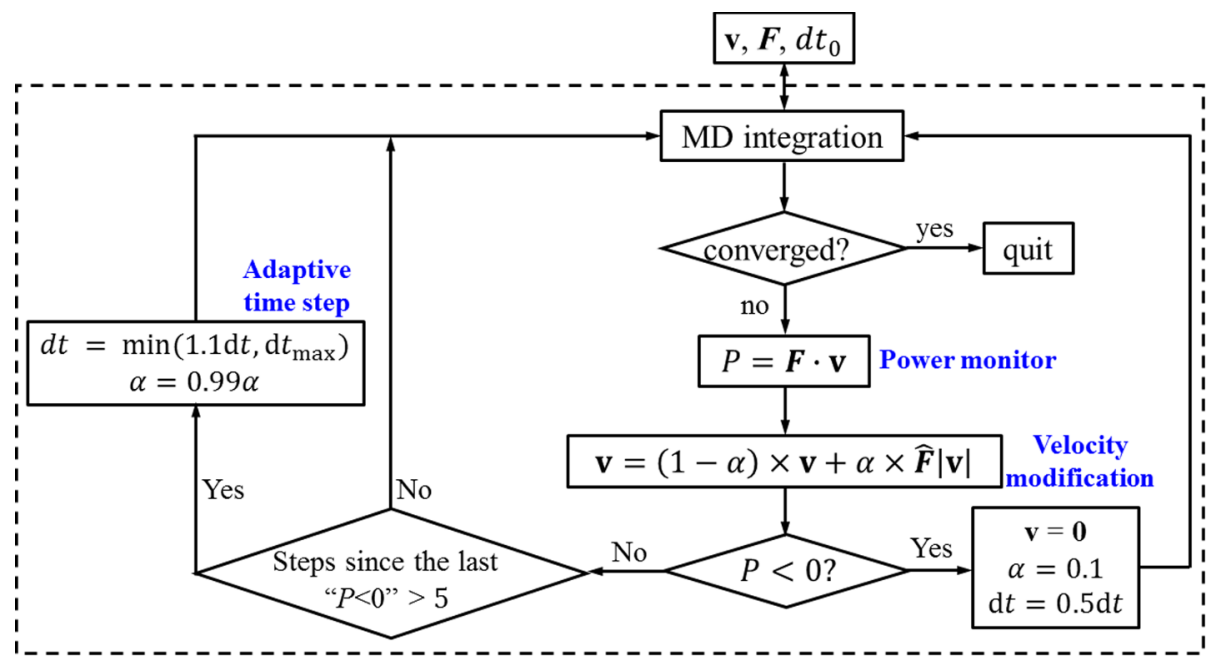

Fig. 1. Computational flow chart of the FIRE method in energy minimization of atomic systems.

Table 1

Details of integration formulations used in the work.

\begin{tabular}{|c|c|c|c|}
\hline Formulations & Position update & Velocity update & Storage \\
\hline Forward Euler (FE) & $x_{n+1}=x_{n}+v_{n} \Delta t$ & $v_{n+1}=v_{n}+a_{n} \Delta t$ & $3 N$ \\
\hline Semi-implicit Euler (SE) & $x_{n+1}=x_{n}+v_{n} \Delta t$ & $v_{n+1}=v_{n}+a_{n+1} \Delta t$ & $3 N$ \\
\hline Velocity Verlet (VV) & $x_{n+1}=x_{n}+v_{n} \Delta t+\frac{1}{2} a_{n} \Delta t^{2}$ & $v_{n+1}=v_{n}+\frac{1}{2}\left(a_{n+1}+a_{n}\right) \Delta t$ & $4 N$ \\
\hline
\end{tabular}

\section{FIRE method and integration algorithms}

Fig. 1 shows the flow chart of FIRE when minimizing the energy of a given system. Like QM, FIRE removes kinetic energy from the given system conditionally to find a local minimal energy state. However, its superiority over QM lies in two key aspects: velocity modification with more inertia effect and adaptive time step by monitoring power state $P$ of the system. In an atomic system, let $\mathbf{x}$ be position vector, $E(\mathbf{x})$ be total potential energy, then $F=-\nabla E(\mathbf{x})$ is atomic force vector; $\mathbf{v}=\dot{\mathbf{x}}$ is velocity vector. The power $P$ is defined as $P=F \cdot v$ and used to monitor whether the system goes toward the lower energy state or not. If $P$ becomes negative, the system will be frozen by setting $\mathbf{v}=0$ to avoid further uphill movement; and time step will be reduced by half in the next iteration. While if $P$ is positive, the system will go further with increasing time step by $10 \%$ so as to promote the convergence rate. The contribution of force in velocity modification, $\alpha$, will also be adjusted automatically during iterations.

The MD integration in Fig. 1 can be performed with different formulations. Three commonly used expressions are Forward Euler (FE), Semi-implicit Euler (SE) and Velocity Verlet (VV) as listed in Table 1. One of the most popular MD simulation packages, LAMMPS [14], takes $\mathrm{FE}$ as the only integrator for FIRE in the latest version. The difference of integration formulations rests on how to update atomistic positions and velocities. FE and SE use forces of the last and current step respectively to update velocities. VV uses velocities and forces of the last step to update positions, and then uses averaged forces of the last and current step to update velocities. Given a system with $N$ atoms, both FE and SE are first-order integrator, and $3 N$ vectors are required to store position, velocity and acceleration data of the current step. $\mathrm{VV}$ is a second-order integrator and needs extra $N$ vectors to store acceleration data of the last step.

In order to explore the influence of integration formulations on the performance of FIRE, three typical minimization systems are investigated: (i) optimization of the two-dimensional (2D) spiral potential energy function, which is the trial problem in the original FIRE paper [13], (ii) relaxation of a $2 \mathrm{D}$ crystal atomic system which is a typical problem in molecular simulations and (iii), propagation of a 2D edge dislocation with atoms jumping over minor energy barriers. Results are discussed in comparison with that of the conventional CG and the limited memory Broyden-Fletcher-Goldfarb-Shanno (LBFGS) methods.

\section{Results and discussions}

\subsection{Spiral-shaped potential energy function}

With a corrugated and intricate landscape, the spiral-shaped potential energy function (Eq. (1) offers a heuristic benchmark to compare different minimization methods. The inset in Fig. 2(a) shows the landscape of the function with the minimum point locating at the center of the "disc", and an initial guess near the edge is selected as the starting point. Fig. 2(a) shows the evolution of the azimuthal angle $\theta$ in Eq. (1) versus the number of function calls of different methods. Obviously, LBFGS is always ahead of CG. FIRE with different integration formulations present huge difference. FIRE using SE takes only 296 function calls to reach the minimum point, which is even faster than LBFGS of 200 function calls. FIRE using VV is slightly slower than using SE, and surpasses LBFGS after 230 function calls. FIRE using FE moves to $\theta$ of only about $\pi / 12$ after 296 functions calls, which is much slower than CG and LBFGS. For the rate of change, SE and VV increase nonlinearly, whereas CG and LBFGS increase linearly. Although FIRE using $\mathrm{SE}$ and VV are slower at the beginning, they show better efficiency than CG and LBFGS overall.

The above difference can also be observed from their trajectories. Fig. 2(b) gives the whole trajectory of SE and the partial trajectories of FE, CG and LBFGS within the dashed box. SE moves almost along the bottom spiral curve smoothly and perfectly, and each function call makes a positive contribution to the minimization process. $\mathrm{VV}$ is roughly close to SE, and the final difference comes from the 296th iteration. Different from the former two integrators, FE moves with random distances and wild directions which often deviates from the bottom spiral curve and goes uphill. Each function call in FE makes less or even negative contribution to the minimization process, so that the efficiency is much worse than that of SE and VV. As for classical minimization methods, LBFGS goes farther than CG in each iteration, 

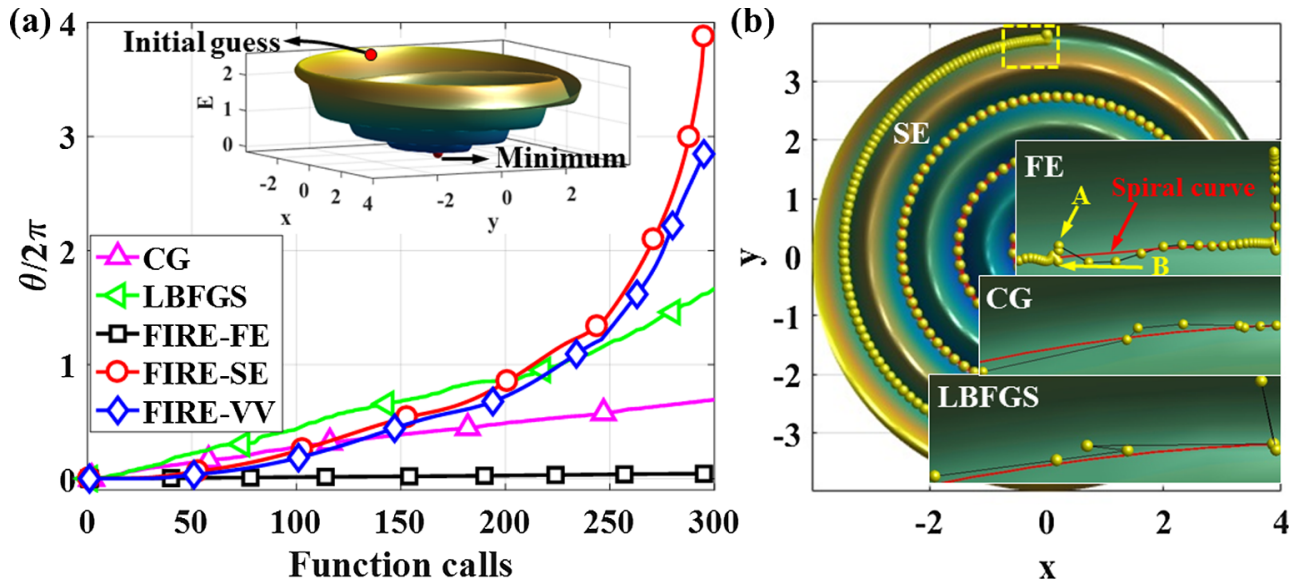

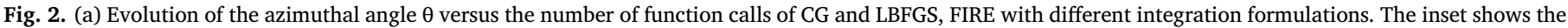
landscape of objective function, initial guess and minimum point. (b) Trajectories of SE, FE, CG and LBFGS.
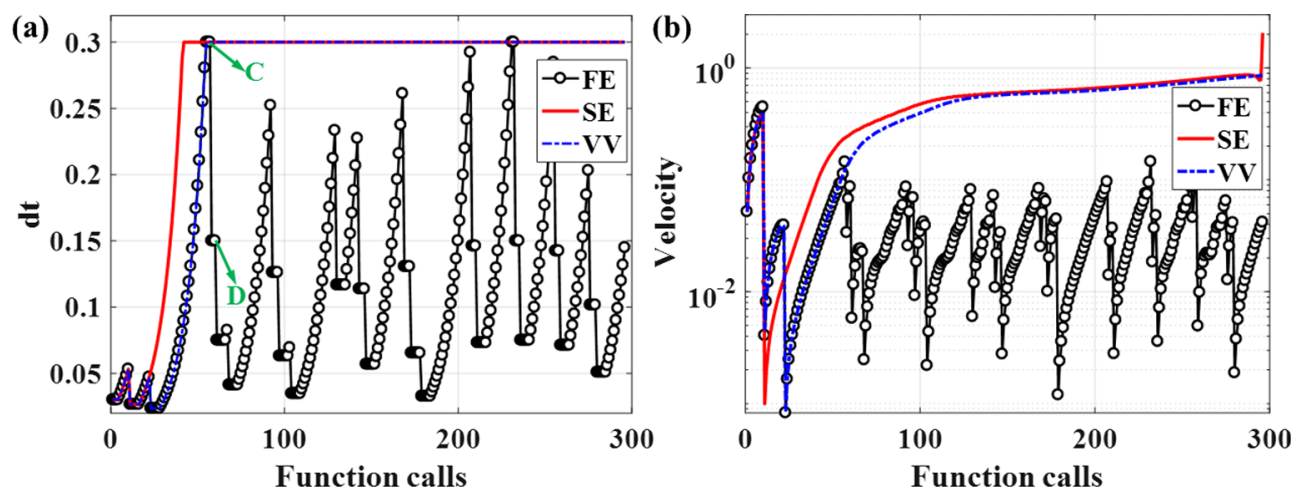

Fig. 3. Time step evolution of the three integration formulations during the optimization of the spiral-shaped energy function.

and both of them deviate from the bottom spiral curve in a certain extent.

$E=[\sin (\pi \theta+r / 2)]^{2}+r^{2} / 10$

Fig. 3(a) presents the evolution of adaptive time step of the three integration formulations. The initial time step is set as 0.03, and FIRE could accelerate the evolution adaptively with maximum time step of 0.3 . The accelerated efficiency $\eta$ is defined as the ratio of iterations with the maximum time step over total iterations. Simulation results show that $\eta=1.69 \%, 86.15 \%$ and $81.76 \%$ for FE, SE and $\mathrm{VV}$ respectively. The three curves coincide with each other at the beginning, and then SE and $\mathrm{VV}$ increase quickly and reach the maximum time step 0.3 after 43 and 55 iterations. No reduction of time step is observed for SE and VV, since their trajectories are very close to the spiral curve. However, FE shows completely different behavior. At the iteration C in Fig. 3, FE drives the system to the point A in Fig. 2(b) where the trial step is far away from the bottom spiral curve. The monitor $P$ becomes negative and the system is frozen. Therefore, time step is reduced by half at the next iteration, and the system stays at point A due to zero velocities. Similarly, iteration at D in Fig. 3(a) corresponds to the other turning point B in Fig. 2(b), and all drops in Fig. 3(a) are corresponding to turning points in Fig. 2(b). Apparently, SE and VV can predict the ongoing path better than $\mathrm{FE}$, and hence results in the better accelerated efficiency.

Compared with CG an LBFGS, inertia effect plays an important role in FIRE to improve its efficiency. From the view of algorithm design, FIRE has more physical meaning than CG and LBFGS. CG and LBFGS use modified gradient and non-exact line search to update positions, while FIRE takes velocity as the whole (SE) or major (VV) contribution when updating positions. In FIRE, inertia can be accumulated gradually during the dynamics evolution if no freezing operation is triggered, like the results of SE and VV in Fig. 3(b). When the accumulated inertia effect prevails over non-exact line search, FIRE using SE and VV will surpass CG and LBFGS in efficiency as shown in Fig. 2(a). In this application, FE is a bad formulation to accumulate inertia as shown in Fig. 3(b). Once the system is frozen, only local forces with tiny time step can be used to update velocities and positions, so it is much slower than CG and LBFGS. Without a sufficient accumulation of inertia, the efficiency of FIRE is not competitive as it would be.

\subsection{Relaxation of atomistic structures}

Relaxation of initial atomistic configuration is a basic application in molecular simulations. A 2D atomistic configuration as shown in Fig. 4 is used to investigate the performance of different minimization methods. The configuration is modeled by 2D square-shaped single crystal with free surfaces as shown in Fig. 4. The initial lattice constant is assigned as $2.5 \AA$. All atoms are colored by individual force norm $\|f\|=\sqrt{f_{x}^{2}+f_{y}^{2}+f_{z}^{2}}$. Before minimization, only the outer layers of atoms are under the non-equilibrium state due to surface effect. The convergence criterion is $\|f\|<1 \times 10^{-10} \mathrm{eV} / \AA$. The L-J potential is used to describe the atomic interaction: $V(r)=4 \varepsilon\left[(\sigma / r)^{12}-(\sigma / r)^{6}\right]$, where $\sigma=2.3276 \AA$ and $\varepsilon=0.4912 \mathrm{eV}$.

Fig. 5(a) shows the evolution of averaged force norm $f_{\text {ave }}=\sum\left\|f_{i}\right\| / N$ versus number of energy evaluation of different minimization methods. Again, FIRE with the three integration formulations exhibits different performance. Specifically, $f_{\text {ave }}$ of FE decreases slowly with frequent fluctuation and small slopes, which has the worst performance among the others. FIRE with SE and VV show superior performance even better than LBFGS and CG. Convergence rate of SE is 
$10.21 \mathrm{~nm}$

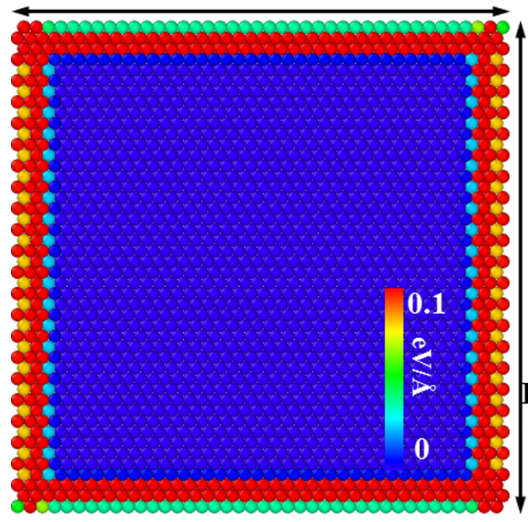

Fig. 4. Atomistic configuration before and after energy minimization (atoms are colored by force norm).
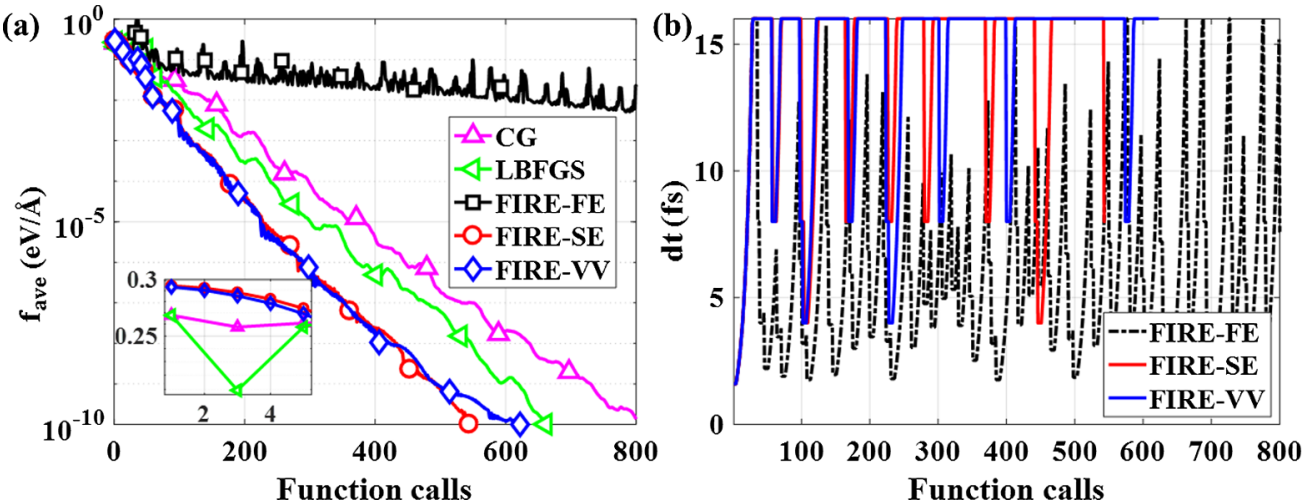

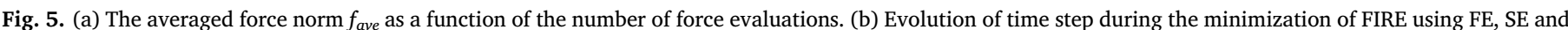
VV integration formulations.

slightly better than that of VV. As mentioned above, the two key features of FIRE method are the introduction of adaptive time step and more inertia effect. The accelerated efficiency of FE, SE and VV is 1.6\%, $74 \%$ and $77 \%$ respectively. FIRE using SE and VV are slower than CG and LBFGS in the first 5 function calls, because inertia effect is not strong enough at the beginning. Although the system is frozen for several times thereafter in SE and VV, few function calls are needed to re-accumulate sufficient inertia to exceed CG and LBFGS, while FE fails to accumulate inertia continuously.

\subsection{Dislocation propagation in atomistic system}

Dislocation evolution and propagation are the fundamental issues in deformation and failure analysis of crystalline materials. In atomistic view, dislocation propagation involves atoms moving between adjacent energy barriers. Here the propagation of a 2D edge dislocation is simulated using different minimization methods to compare their performance. As shown in Fig. 6, an initial dislocation is inserted into a 2D single crystal slab by displacing atoms according to the elastic displacement field around an edge dislocation core [23]. The top and bottom layers of atoms are fixed to provide constraints. A simple shear loading is applied to drive the edge dislocation as illustrated in Fig. 6. The convergence criterion is $f_{\text {ave }}<10^{-4} \mathrm{eV} / \mathrm{A}$. The atomic potential is the same as that described in Section 3.2.

Minimization process in this case can be divided into two stages. Firstly, the potential energy decreases gradually as the dislocation moves to the right end and secondly, the system approaches to a steady state with slight decreases due to local adjustment of atomic positions after the dislocation disappears or stops. When the applied shear strain is increased to 0.03 , the dislocation moves to the right end and a slip

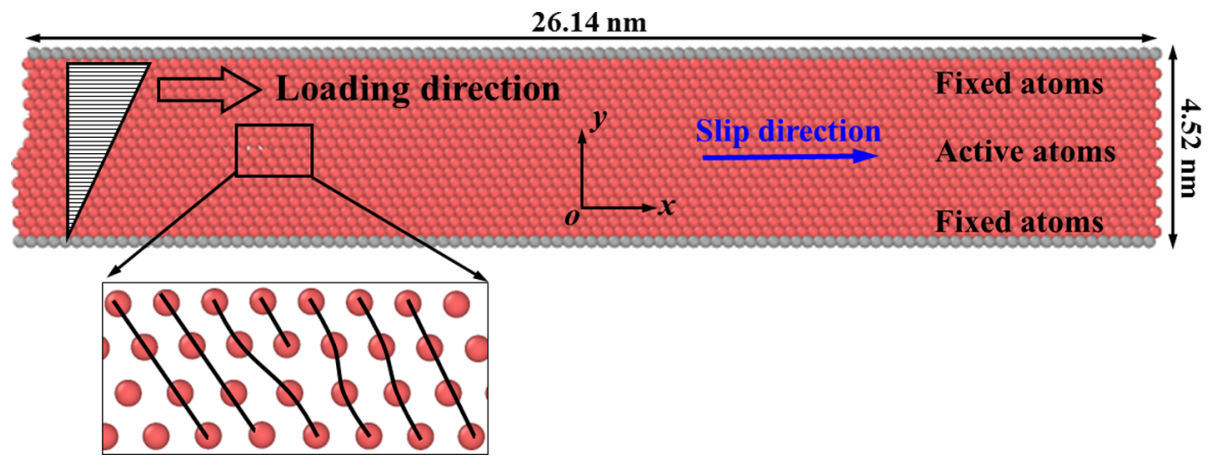

Fig. 6. Atomistic model for simulations of edge dislocation propagation. 

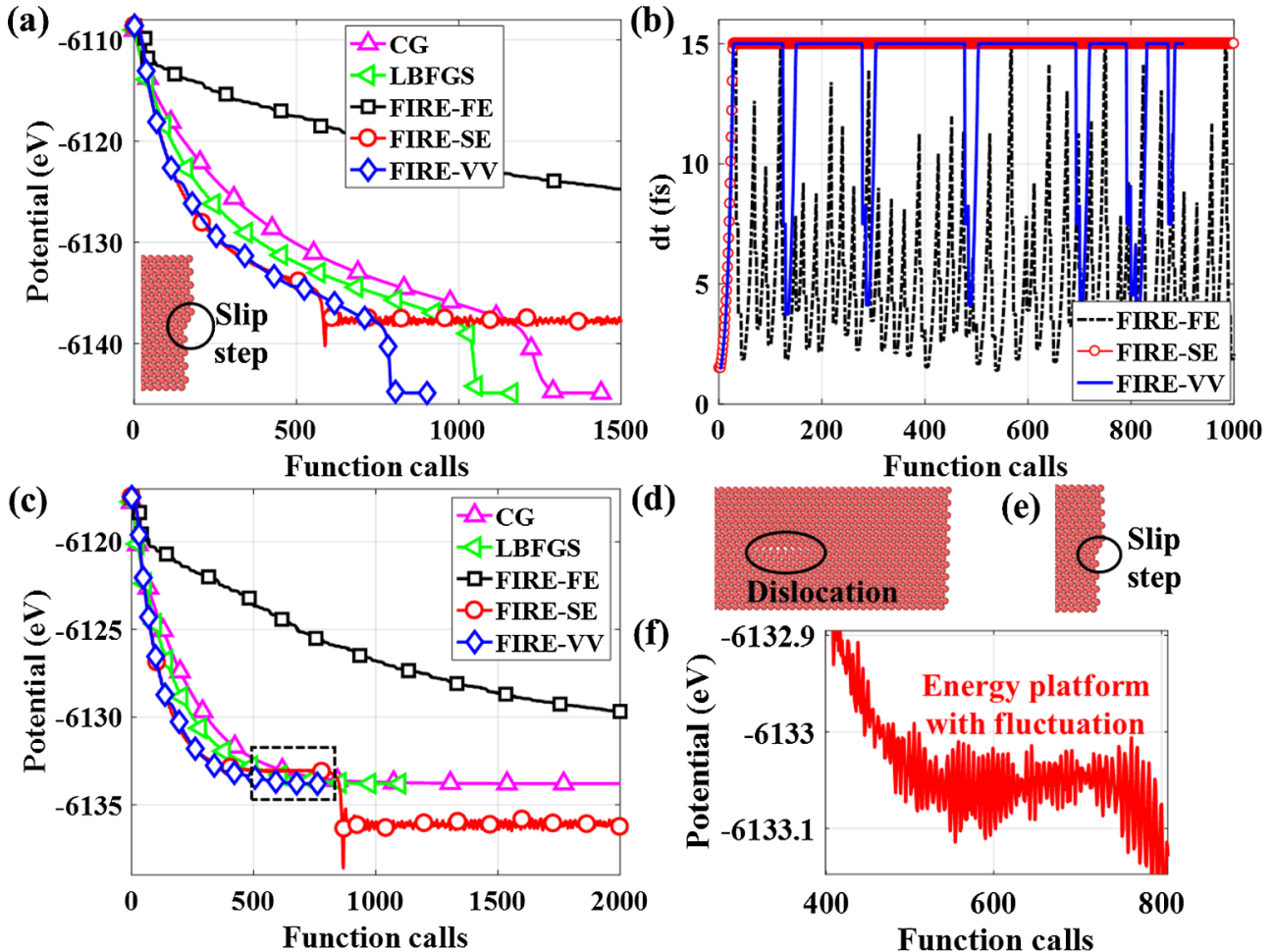

(d)

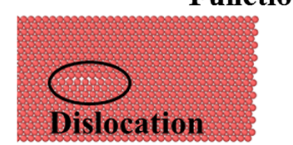

(e)

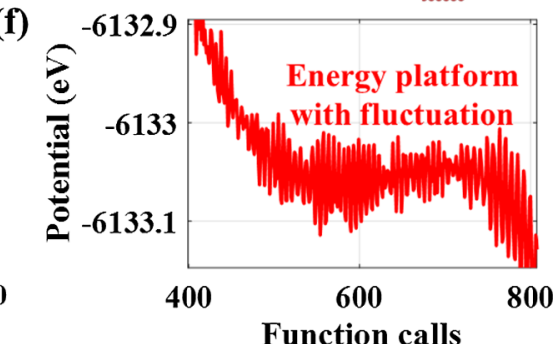

Fig. 7. Evolution of system energy in minimization using CG, LBFGS and FIRE under shear strain 0.03 (a) and 0.025 (c). (b) The evolution of time step during FIRE minimization using FE, SE and VV integration formulations. (d) Trapped dislocation using CG, LBFGS and FIRE-VV. (e) Slip step formed by FIRE-SE when the shear strain is 0.025. (f) Minor energy barriers FIRE-SE passes when the shear strain is 0.025 . step is formed, as shown in the inset of Fig. 7(a). As expected, LBFGS is faster than CG like in the former two cases, and both of them can converge to a low energy state of $-6145 \mathrm{eV}$. For the different integrators in FIRE method, FE is still the worst one, while SE and VV finish the first stage with only 580 and 800 function calls respectively. However, the iteration of SE is not terminated in the second stage, as the system energy vibrates around a higher value of $-6137 \mathrm{eV}$. The convergence criterion of $\mathrm{VV}$ is met after 100 function calls when the dislocation disappears, which is faster than LBFGS. According to the evolution of time step in Fig. 7(b), accelerated efficiency $\eta$ are calculated as $0.7 \%, 98.6 \%$ and $79.7 \%$ for FE, SE and VV respectively. Since the potential landscape in dislocation evolution is more complicated than that in 2D spiral function and structure relaxation, accelerated efficiency of FE is smaller than that of the other two cases. SE is the fastest in the first stage, but the uninterrupted acceleration gives no chance to extract kinetic energy from the system, which leads to ceaseless vibration of energy in the second stage. Therefore, VV is the only integration formulation that makes FIRE better than LBFGS and CG in this case.

FIRE has the direct ability to critical analysis [13], i.e., a class of typical relaxation problems used to determine saddle points, transition states or critical points, which is not possible with some conventional EM algorithms like CG and LBFGS due to their strict adherence to minimizing forces. The study of mechanical stability, like calculating the Peierls stress of a dislocation or determining a transition state, is a common task of critical analysis in computational material sciences. For example, EM was used to calculate the pressure dependence of the Peierls stress in Aluminum [24]. In order to explore the influence of integrator on the ability, the shear strain is decreased to 0.025. As shown in Fig. 7(c), FIRE-FE is still the worst one. FIRE-VV, CG and LBFGS converge to the same energy of $-6133.5 \mathrm{eV}$ successively, but the dislocation is trapped into the system as shown in Fig. 7(d). Only FIRE-SE can decrease the energy further to $-6136.5 \mathrm{eV}$ after the dislocation passes through an energy platform with minor barriers as shown in Fig. 7(f). The dislocation moves to the right end and a slip step is formed (Fig. 7(e)). SE can drive the system to go through a shallow valley with very small forces that FIRE-VV, CG and LBFGS cannot.
Nevertheless, SE is still unable to converge. Therefore, the ability of critical analysis of FIRE is dependent on integration formulations. In this application, only SE endows FIRE with the ability of critical analysis, but its convergence cannot be guaranteed.

The results from different integration formulations in Fig. 7 can be understood by analyzing the atomic trajectories in Fig. 8 when the shear strain is 0.03 . Propagation of a dislocation happens by the repeat of the breaking and formation of atomic bonds near the dislocation core. As shown in Fig. 8(a), the atom 1 moves along a convex parabolalike curve from $\mathrm{A}$ to $\mathrm{B}$, while the atom 2 moves along a concave parabola-like curve in the opposite direction, which constitutes the basic propagation unit of the dislocation. The shape of trajectories depends on minimization methods. The trajectory of FE is close to a symmetry parabola. Curves of SE and VV are thinner and taller, bend to the glide direction and coincide with each other except at the right tail. The atom 1 in SE dangles at the right tail of the trajectory which leads to the endless fluctuation of the potential energy as shown in Fig. 7(a). The trajectories of CG and LBFGS show similar features of SE and VV, but deviate from each other at the top of the curves. Fig. 8(b) gives enlarged trajectories of dashed box labeled in Fig. 8(a). The curve of FE is rough due to frequent freezing operations, while SE and VV are much smoother. Besides, the atom 1 in SE moves with larger distance than that in VV, which gives SE the ability to jump minor energy barriers. Compared with CG and LBFGS, the smoother curves of SE and VV indicate that FIRE can predict lower energy path with proper integration formulations. The transition path of dislocation evolution in Fig. 8 shows the connection between minimization algorithm and physical meaning which may be helpful for designing new efficient minimization methods.

\subsection{Role of monitor}

As discussed above, FE is ill-suited for all the three cases; SE shows the best efficiency but cannot reach the minimal state in the case of dislocation propagation; and VV requires higher memory usage. The role of monitor is investigated here to make FIRE more favorable for energy minimization. It is interesting that both FIRE and QM use power 

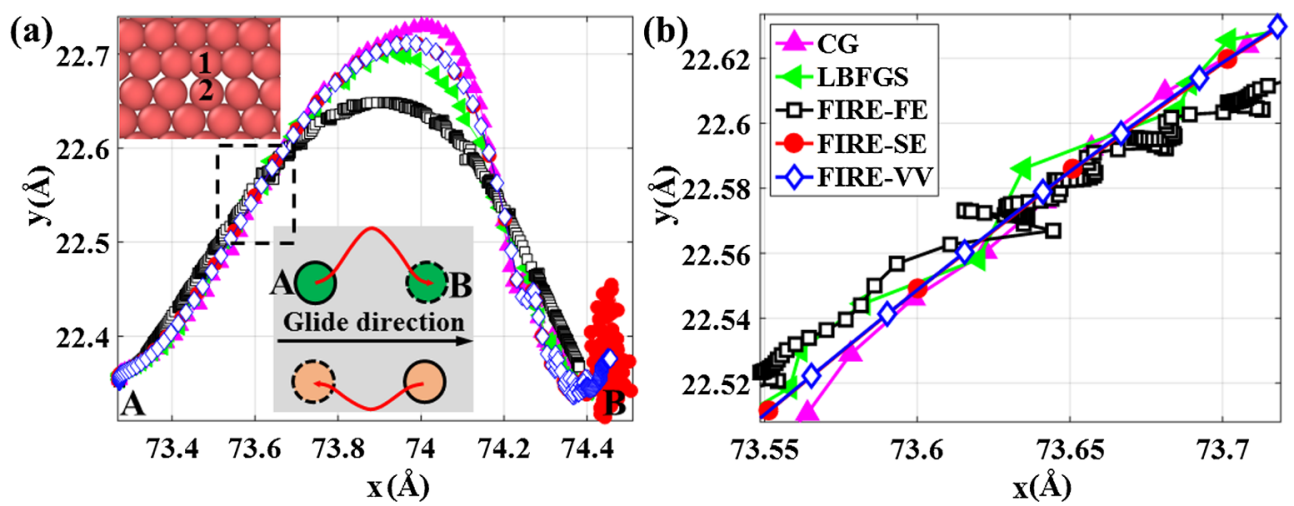

Fig. 8. (a) Trajectories of the atom 1 in the dislocation core obtained from different methods. (b) Enlarged trajectories in the dashed box of (a).
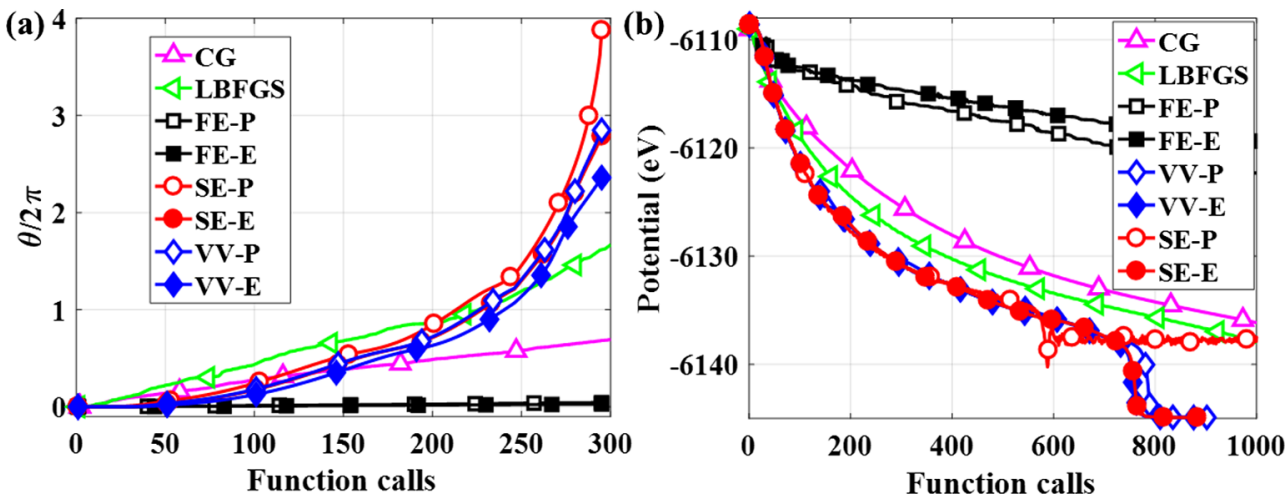

Fig. 9. (a) Optimization of spiral-shaped energy function and (b) dislocation propagation using FIRE with different integrators and monitors.

$P$ rather than energy change as monitor in minimization. For a conservative system, a positive power $P$ indicates that energy is transferring from potential to kinetic, i.e., the potential energy decreases, so the power monitor should be equivalent to energy monitor. However, the velocity modification and discrete numerical integration in Fig. 1 break energy conservation and lead to increase of potential energy for positive $P$, as shown in Fig. 7(a) and (c). Compared with power monitor, we found that energy monitor is more direct and stricter in energy minimization, thus it can be used to limit acceleration efficiency and assure convergence of FIRE.

The energy monitor is defined as the energy change $\Delta E=E_{\text {new }}-E_{\text {old }}$ and replaces power monitor in Fig. 1. If $\triangle E<0$, the accelerated dynamics is used, otherwise the system is frozen by setting all velocities to zero. Fig. 9(a) shows the minimization results of the 2D spiral potential function using different methods. For FE, no obvious change is observed between energy and power monitor. SE and VV with energy monitor are slightly slower than that with power monitor, but still faster than LBFGS and CG. Fig. 9(b) shows the evolution of potential energy during the dislocation propagation at strain of 0.03 obtained from different methods. Changing to energy monitor has little influence on the performance of $\mathrm{VV}$, but restrains the vibration of SE and assures its convergence. Therefore, the energy monitor imposes stricter control than power monitor, and makes SE behave like VV. Considering memory usage, SE with energy monitor should be the better choice for FIRE in general MS simulations. Nevertheless, SE with power monitor is the only option for critical analysis.

\section{Summary}

FIRE method with three commonly used integrators (FE, SE and VV), CG and LBFGS are applied to three typical energy minimization problems to investigate the influence of integration formulations on the performance of FIRE. The results indicate that efficiency, convergence and the ability of critic analysis of FIRE are strongly dependent on integration formulation which is different from the viewpoint of the FIRE authors. Among these EM methods, FIRE-FE is ill-suited for all the trial applications, and always leads to terrible efficiency and convergence performance. Aggressive SE endows FIRE with good efficiency even higher than CG and LBFGS. FIRE-SE also shows the capability of critical analysis, but its convergence is conditional. Fortunately, a stricter energy monitor is found effective to make SE converge. FIRE-VV is a moderate strategy and shows good efficiency and convergence in all cases, but it is not suitable for critical analysis. Generally, based on the results, SE combined with energy monitor shows superior convergence and should be the first choice for MS simulations when using FIRE method. However, for critical analysis, FIRE-SE along with power monitor should be used.

\section{CRediT authorship contribution statement}

The manuscript was written based on the contributions made by all authors (Fei Shuang, Pan Xiao, Ronghao Shi, Fujiu Ke and Yilong Bai): Pan Xiao: devised the project, the main conceptual ideas and proof outline. Fei Shuang: worked out almost all of the technical details, and performed most of the numerical calculations. Ronghao Shi: performed partial of the MD simulations. Yilong Bai and Fujiu Ke: were involved in all discussions and problem solving in the simulations.

\section{Acknowledgement}

Support from the Strategic Priority Research Program (B) of the Chinese Academy of Sciences (XDB22040501), the National Natural Science Foundation of China (Grant nos. 11672298, 11432014 and 11790291), is gratefully acknowledged. Computations are performed 
on the ScGrid of Supercomputing Center, Computer Network Information Center of Chinese Academy of Sciences.

\section{References}

[1] O. Vinogradov, A new method of molecular statics in polycrystals applications, Comput. Mater. Sci. 39 (2007) 611-615, https://doi.org/10.1016/j.commatsci. 2006.08.019.

[2] F. Muñoz, J. Rogan, J.A. Valdivia, A. Varas, M. Kiwi, Binary cluster collision dynamics and minimum energy conformations, Phys. B Condens. Matter. 427 (2013) 76-84, https://doi.org/10.1016/j.physb.2013.06.036.

[3] D. Bhattacharya, J. Cheng, 3Drefine: Consistent protein structure refinement by optimizing hydrogen bonding network and atomic-level energy minimization, Proteins Struct. Funct. Bioinforma. 81 (2013) 119-131, https://doi.org/10.1002/ prot. 24167.

[4] S. Groh, E.B. Marin, M.F. Horstemeyer, D.J. Bammann, Dislocation motion in magnesium: a study by molecular statics and molecular dynamics, Model. Simul. Mater. Sci. Eng. 17 (2009) 075009, https://doi.org/10.1088/0965-0393/17/7/ 075009.

[5] G. Settanni, J. Zhou, T. Suo, S. Schöttler, K. Landfester, F. Schmid, V. Mailänder, Protein corona composition of PEGylated nanoparticles correlates strongly with amino acid composition of protein surface, (2016). 10.1039/x0xx00000x.

[6] B. Eidel, A. Stukowski, J. Schröder, Energy-minimization in atomic-to-continuum scale-bridging methods, PAMM. 11 (2011) 509-510, https://doi.org/10.1002/ pamm.201110246.

[7] P. Zhang, Z. Ma, Y. Wang, Y. Zou, W. Lei, Y. Pan, C. Lu, A first principles study of the mechanical properties of Li-Sn alloys, RSC Adv. 5 (2015) 36022-36029, https:// doi.org/10.1039/c5ra04685h.

[8] P. Zhang, Z. Ma, W. Jiang, Y. Wang, Y. Pan, C. Lu, Mechanical properties of Li-Sn alloys for Li-ion battery anodes: a first-principles perspective, AIP Adv. 6 (2016), https://doi.org/10.1063/1.4940131.

[9] J. Chen, P. Ming, An efficient multigrid method for molecular mechanics modeling in atomic solids, Commun. Comput. Phys. 10 (2011) 70-89, https://doi.org/10. 4208/cicp.270910.131110a.

[10] J. Chen, P. Ming, J.Z. Yang, A constrained Cauchy-Born elasticity accelerated multigrid method for nanoindentation, Commun. Comput. Phys. 15 (2014) 470-486, https://doi.org/10.4208/cicp.020912.150713a.

[11] J. Chen, C.J. García-Cervera, An efficient multigrid strategy for large-scale molecular mechanics optimization, J. Comput. Phys. 342 (2017) 29-42, https://doi.org/ 10.1016/j.jcp.2017.04.035.

[12] D. Sheppard, R. Terrell, G. Henkelman, Optimization methods for finding minimum energy paths, J. Chem. Phys. 128 (2008) 134106, https://doi.org/10.1063/1. 2841941.

[13] E. Bitzek, P. Koskinen, F. Gähler, M. Moseler, P. Gumbsch, Structural relaxation made simple, Phys. Rev. Lett. 97 (2006) 170201, https://doi.org/10.1103/ PhysRevLett. 97.170201.

[14] S. Plimpton, Fast parallel algorithms for short-range molecular dynamics, J. Comput. Phys. 117 (1995) 1-19, https://doi.org/10.1006/jcph.1995.1039.

[15] J. Glaser, T.D. Nguyen, J.A. Anderson, P. Lui, F. Spiga, J.A. Millan, D.C. Morse, S.C. Glotzer, Strong scaling of general-purpose molecular dynamics simulations on GPUs, Comput. Phys. Commun. 192 (2015) 97-107, https://doi.org/10.1016/j.cpc. 2015.02.028.

[16] J.A. Anderson, C.D. Lorenz, A. Travesset, General purpose molecular dynamics simulations fully implemented on graphics processing units, J. Comput. Phys. 227 (2008) 5342-5359, https://doi.org/10.1016/j.jcp.2008.01.047.

[17] L. Genovese, A. Neelov, S. Goedecker, T. Deutsch, S.A. Ghasemi, A. Willand, D. Caliste, O. Zilberberg, M. Rayson, A. Bergman, R. Schneider, Daubechies wavelets as a basis set for density functional pseudopotential calculations, J. Chem. Phys. 129 (2008), https://doi.org/10.1063/1.2949547.

[18] S.T. Chill, M. Welborn, R. Terrell, L. Zhang, J.C. Berthet, A. Pedersen, H. Jónsson, G. Henkelman, EON: software for long time simulations of atomic scale systems, Model. Simul. Mater. Sci. Eng. 22 (2014), https://doi.org/10.1088/0965-0393/22/ 5/055002.

[19] J. Rogan, A. Varas, J.A. Valdivia, M. Kiwi, A strategy to find minimal energy nanocluster structures, J. Comput. Chem. 34 (2013) 2548-2556, https://doi.org/10. 1002/jcc.23419.

[20] P. Fayon, A. Trewin, Formation mechanism of ultra porous framework materials, Phys. Chem. Chem. Phys. 18 (2016) 16840-16847, https://doi.org/10.1039/ c6cp02764d.

[21] H.J. Hwang, R.A. Riggleman, J.C. Crocker, Understanding soft glassy materials using an energy landscape approach, Nat. Mater. 15 (2016) 1031-1036, https:// doi.org/10.1038/nmat4663.

[22] L. Yang, C. Hou, X. Ma, L. Ye, L. Chang, L. Shi, X. He, Structure relaxation via long trajectories made stable, Phys. Chem. Chem. Phys. 19 (2017) 24478-24484, https://doi.org/10.1039/c7cp04838f.

[23] A.H. Cottrell, Theory of dislocations, Prog. Met. Phys. 4 (1953) 205-264, https:// doi.org/10.1016/0502-8205(53)90018-5.

[24] K. Dang, D. Spearot, Pressure dependence of the peierls stress in aluminum, JOM 70 (2018) 1094-1099, https://doi.org/10.1007/s11837-018-2819-y. 